\title{
Spectral Bounds for Polydiagonal Jacobi Matrix Operators
}

\author{
Arman Sahovic \\ Mathematics Department, Imperial College London, South Kensington, 180 Queen's Gate, London SW7 2AZ, UK \\ Correspondence should be addressed to Arman Sahovic; arman@ic.ac.uk
}

Received 24 April 2014; Accepted 4 September 2014; Published 19 October 2014

Academic Editor: Michael Grinfeld

Copyright (C) 2014 Arman Sahovic. This is an open access article distributed under the Creative Commons Attribution License, which permits unrestricted use, distribution, and reproduction in any medium, provided the original work is properly cited.

\begin{abstract}
The research on spectral inequalities for discrete Schrödinger operators has proved fruitful in the last decade. Indeed, several authors analysed the operator's canonical relation to a tridiagonal Jacobi matrix operator. In this paper, we consider a generalisation of this relation with regard to connecting higher order Schrödinger-type operators with symmetric matrix operators with arbitrarily many nonzero diagonals above and below the main diagonal. We thus obtain spectral bounds for such matrices, similar in nature to the Lieb-Thirring inequalities.
\end{abstract}

\section{Background}

Let $W$ be the self-adjoint Jacobi matrix operator acting on $\ell^{2}(\mathbb{Z})$ as follows:

$$
W=\left(\begin{array}{cccccc}
\ddots & \vdots & \vdots & \vdots & \vdots & . \\
\ldots & b_{-1} & a_{-1} & 0 & 0 & \ldots \\
\ldots & a_{-1} & b_{0} & a_{0} & 0 & \ldots \\
\ldots & 0 & a_{0} & b_{1} & a_{1} & \ldots \\
\ldots & 0 & 0 & a_{1} & b_{2} & \ldots \\
. & \vdots & \vdots & \vdots & \vdots & \ddots
\end{array}\right)
$$

via

$$
\begin{array}{r}
(W \varphi)(n)=a_{n-1} \varphi(n-1)+b_{n} \varphi(n)+a_{n} \varphi(n+1), \\
\text { for } n \in \mathbb{Z},
\end{array}
$$

where $a_{n}>0$ and $b_{n} \in \mathbb{R}$. This operator can be viewed as the one-dimensional discrete Schrödinger operator if $a_{n}=$ 1 for all $n$. A variety of papers examined such operators; for example, we quote the work by Killip and Simon in [1], where they obtained sum rules for such Jacobi matrices. Additionally, Hundertmark and Simon in [2] were able to find spectral bounds for these operators. We thus state their result.

If $a_{n} \rightarrow 1, b_{n} \rightarrow 0$ rapidly enough, as $n \rightarrow \pm \infty$, the essential spectrum $\sigma_{\text {ess }}(W)$ of $W$ is absolutely continuous and coincides with the interval $[-2,2]$ (see, e.g., [3]). Besides, $W$ may have simple eigenvalues $\left\{E_{j}^{ \pm}\right\}_{j=1}^{N_{ \pm}}$where $N_{ \pm} \in \overline{\mathbb{N}}(:=\mathbb{N} \cup$ $\{\infty\})$, and

$$
E_{1}^{+}>E_{2}^{+}>\cdots>2>-2>\cdots>E_{2}^{-}>E_{1}^{-} \text {. }
$$

Indeed, in [2] the authors found the following.

Theorem 1. If $\left\{b_{n}\right\}_{n \in \mathbb{Z}},\left\{a_{n}-1\right\}_{n \in \mathbb{Z}} \in \ell^{\gamma+1 / 2}(\mathbb{Z}), \gamma \geq 1 / 2$, then

$$
\begin{aligned}
& \sum_{j=1}^{N_{+}}\left|E_{j}^{+}-2\right|^{\gamma}+\sum_{j=1}^{N_{-}}\left|E_{j}^{-}+2\right|^{\gamma} \\
& \quad \leq k_{\gamma}\left[\sum_{n=-\infty}^{\infty}\left|b_{n}\right|^{\gamma+1 / 2}+4 \sum_{n=-\infty}^{\infty}\left|a_{n}-1\right|^{\gamma+1 / 2}\right],
\end{aligned}
$$

where

$$
k_{\gamma}=2\left(3^{\gamma-1 / 2}\right) L_{\gamma, 1}^{c l}, \quad L_{\gamma, 1}^{c l}=\frac{\Gamma(\gamma+1)}{2 \sqrt{\pi} \Gamma(\gamma+3 / 2)} .
$$

The author (see [4]) then improved their result, achieving the smaller constant: $k_{\gamma}=3^{\gamma-1} \pi L_{\gamma, 1}^{c l}$, by translating a wellknown method employed by Dolbeaut et al. in [5] to the discrete scenario. They, in turn, used a simple argument by Eden and Foias (see [6]) to obtain improved constants for Lieb-Thirring inequalities in one dimension.

The aim of this paper is to answer the natural question of whether these methods can be generalised to give bounds 
for higher order Schrödinger-type operators and thus "polydiagonal" Jacobi-type matrix operators, which we will define below.

\section{Notation and Preliminary Material}

For a sequence $\{\varphi(n)\}_{n \in \mathbb{Z}}$, let $D$ and $D^{*}$ be the difference operator and its adjoint, respectively, denoted by $D \varphi(n)=$ $\varphi(n+1)-\varphi(n)$ and $D^{*} \varphi(n)=\varphi(n-1)-\varphi(n)$. We then denote the discrete one-dimensional Laplacian by $\Delta_{D}:=$ $\left(D^{*} D \varphi\right)(n)=-\varphi(n+1)+2 \varphi(n)-\varphi(n-1)$. For $\sigma \in \mathbb{N}, n \in \mathbb{Z}$, and a sequence $\varphi \in \ell^{2}(\mathbb{Z})$, with $\Delta_{D}^{1}=\Delta_{D}$, we define $\Delta_{D}^{\sigma}$ by

$$
\left(\Delta_{D}^{\sigma} \varphi\right)(n):=\left(\Delta_{D}\left(\Delta_{D}^{\sigma-1} \varphi\right)\right)(n) .
$$

We note that $\Delta_{D}$ being self-adjoint immediately implies that $\Delta_{D}^{\sigma}$ is also self-adjoint.

Finding an explicit formula for $\Delta_{D}^{\sigma}$ requires a few combinatorial techniques, all of which are standard. Let ${ }^{a} C_{b}:=$ $\left(\begin{array}{l}a \\ b\end{array}\right):=a ! /((a-b) ! b !)$, for $a, b \in \mathbb{N}$. Then we have the following: (i) ${ }^{a} C_{b}+{ }^{a} C_{b+1}={ }^{a+1} C_{b+1}$, (ii) $2{ }^{a} C_{0}+{ }^{a} C_{1}={ }^{a+2} C_{1}$, and (iii) $2{ }^{a} C_{a}+{ }^{a} C_{a-1}={ }^{a+2} C_{a+1}$.

A simple induction argument then delivers our formula for the $\sigma$ th order discrete Laplacian operator as follows:

$$
\left(\Delta_{D}^{\sigma} \varphi\right)(n)=\sum_{k=0}^{2 \sigma}{ }^{2 \sigma} C_{k}(-1)^{k+\sigma} \varphi(n-\sigma+k) .
$$

Furthermore, in order to identify our essential spectrum, we apply the discrete fourier transform as follows:

$$
\mathscr{F}\left(\Delta_{D}^{\sigma} \varphi\right)(x)=\sum_{n \in \mathbb{Z}} e^{i n x}\left(\sum_{k=0}^{2 \sigma}{ }^{2 \sigma} C_{k}(-1)^{k+\sigma} \varphi(n-\sigma+k)\right),
$$

which, after some rearrangement, yields

$$
\begin{aligned}
& \mathscr{F}\left(\Delta_{D}^{\sigma} \varphi\right)(x) \\
&=\left[{ }^{2 \sigma} C_{\sigma}+2 \sum_{k=0}^{\sigma-1}{ }^{2 \sigma} C_{k}(-1)^{k+\sigma} \cos ((\sigma-k) x)\right] \\
& \times(\mathscr{F} \varphi)(x) .
\end{aligned}
$$

The essential spectrum of the operator $\Delta_{D}^{\sigma}$ will thus be the range of the above symbol, which can be found to be $\varsigma_{\text {ess }}\left(\Delta_{D}^{\sigma}\right)=\left[0,4^{\sigma}\right]$.

\section{Main Results}

We now let $\left\{\psi_{j}\right\}_{j=1}^{N}, N \in \overline{\mathbb{N}}$, be the orthonormal system of eigensequences in $\ell^{2}(\mathbb{Z})$ corresponding to the negative eigenvalues $\left\{e_{j}\right\}_{j=1}^{N}$ of the $(2 \sigma)$ th order discrete Schrödingertype operator as follows:

$$
\left(H_{D}^{\sigma} \psi_{j}\right)(n):=\left(\Delta_{D}^{\sigma} \psi_{j}\right)(n)-b_{n} \psi_{j}(n)=e_{j} \psi_{j}(n),
$$

where $j \in\{1, \ldots, N\}$ and we assume that $b_{n} \geq 0$ for all $n \in \mathbb{Z}$. Our next result is concerned with estimating those negative eigenvalues.
Theorem 2. Let $b_{n} \geq 0,\left\{b_{n}\right\}_{n \in \mathbb{Z}} \in e^{\gamma+1 / 2 \sigma}(\mathbb{Z}), \gamma \geq 1$. Then the negative eigenvalues $\left\{e_{j}\right\}_{j=1}^{N}$ of the operator $H_{D}^{\sigma}$ satisfy the inequality

$$
\sum_{j=1}^{N}\left|e_{j}\right|^{\gamma} \leq \eta_{\sigma}^{\gamma} \sum_{n \in \mathbb{Z}} b_{n}^{\gamma+1 / 2 \sigma}
$$

where

$$
\eta_{\sigma}^{\gamma}:=\frac{2 \sigma}{(2 \sigma+1)^{(2 \sigma+1) / 2 \sigma}} \frac{\Gamma((4 \sigma+1) / 2 \sigma) \Gamma(\gamma+1)}{\Gamma(\gamma+(2 \sigma+1) / 2 \sigma)} .
$$

Remark 3. As the discrete spectrum of $H_{D}^{\sigma}$ lies in $[-\infty, 0]$ and $\left[4^{\sigma}, \infty\right]$, we shift our operator to the left by $4^{\sigma}$ and by analogy have an estimate for the positive eigenvalues of that operator, thus immediately obtaining Corollary 4.

Corollary 4. Let $b_{n} \geq 0,\left\{b_{n}\right\}_{n \in \mathbb{Z}} \in e^{\gamma+1 / 2 \sigma}(\mathbb{Z}), \gamma \geq 1$. Then the positive eigenvalues $\left\{e_{j}\right\}_{j=1}^{N}$ of the operator $\Delta_{D}^{\sigma}-4^{\sigma}+b$ satisfy the inequality:

$$
\sum_{j=1}^{N} e_{j}^{\gamma} \leq \eta_{\sigma}^{\gamma} \sum_{n \in \mathbb{Z}} b_{n}^{\gamma+1 / 2 \sigma}, \text { with } \eta_{\sigma}^{\gamma} \text { given above. }
$$

Finally we will apply these results to obtain spectral bounds for the following operator.

We let $W_{\sigma}$ be a polydiagonal self-adjoint Jacobi-type matrix operator as follows:

$$
W_{\sigma}:=\left(\begin{array}{ccccccccc}
\ddots & \ddots & \ddots & \ddots & \ddots & \ddots & \ddots & \ddots & \ddots \\
\ddots & \ddots & \ddots & \ddots & \ddots & a_{-2}^{\sigma} & 0 & 0 & \ddots \\
\ddots & \ddots & b_{-1} & a_{-1}^{1} & \ddots & \ddots & a_{-1}^{\sigma} & 0 & \ddots \\
\ddots & \ddots & a_{-1}^{1} & b_{0} & a_{0}^{1} & \ddots & \ddots & a_{0}^{\sigma} & \ddots \\
\ddots & \ddots & \ddots & a_{0}^{1} & b_{1} & a_{1}^{1} & \ddots & \ddots & \ddots \\
\ddots & a_{-2}^{\sigma} & \ddots & \ddots & a_{1}^{1} & b_{2} & a_{2}^{1} & \ddots & \ddots \\
\ddots & 0 & a_{-1}^{\sigma} & \ddots & \ddots & a_{2}^{1} & b_{3} & \ddots & \ddots \\
\ddots & 0 & 0 & a_{0}^{\sigma} & \ddots & \ddots & \ddots & \ddots & \ddots \\
\ddots & \ddots & \ddots & \ddots & \ddots & \ddots & \ddots & \ddots & \ddots
\end{array}\right),
$$

viewed as an operator acting on $\ell^{2}(\mathbb{Z})$ as follows: for $n \in \mathbb{Z}$, $i \in\{1, \ldots, \sigma\}$,

$$
\begin{aligned}
\left(W_{\sigma} \varphi\right)(n)= & \sum_{i=1}^{\sigma} a_{n-i}^{i} \varphi(n-i)+b_{n} \varphi(n)+\sum_{i=1}^{\sigma} a_{n}^{i} \varphi(n+i) \\
= & a_{n-\sigma}^{\sigma} \varphi(n-\sigma)+\cdots+a_{n-1}^{1} \varphi(n-1) \\
& +b_{n} \varphi(n)+a_{n}^{1} \varphi(n+1)+\cdots+a_{n}^{\sigma} \varphi(n+\sigma),
\end{aligned}
$$

where $a_{n}^{i}, b_{n} \in \mathbb{R}$, for all $i \in\{1, \ldots, \sigma\}$. We denote $\left(W_{\sigma}\left(\left\{a_{n}^{1}\right\}\right.\right.$, $\left.\left.\ldots,\left\{a_{n}^{\sigma}\right\},\left\{b_{n}\right\}\right) \varphi\right)(n):=\left(W_{\sigma} \varphi\right)(n)$ where we understand $\{\cdot\}$ to mean $\{\cdot\}_{n \in \mathbb{Z}}$. We are then interested in perturbations of the following special case:

$$
\begin{gathered}
\left(W_{\sigma}^{0} \varphi\right)(n):=\left(W _ { \sigma } \left(\left\{a_{n}^{1} \equiv \omega_{1}\right\}, \ldots,\left\{a_{n}^{\sigma} \equiv \omega_{\sigma}\right\},\right.\right. \\
\left.\left.\left\{b_{n} \equiv 0\right\}\right) \varphi\right)(n),
\end{gathered}
$$


where $\omega_{i}:={ }^{2 \sigma} C_{\sigma+i}(-1)^{i}$, and explicitly

$$
\begin{aligned}
\left(W_{\sigma}^{0} \varphi\right)(n) & =\left(\left(\Delta_{D}^{\sigma}-{ }^{2 \sigma} C_{\sigma}\right) \varphi\right)(n) \\
& =\sum_{k=0, k \neq \sigma}^{2 \sigma}{ }^{2 \sigma} C_{k}(-1){ }^{k+\sigma} \varphi(n-\sigma+k),
\end{aligned}
$$

called the free Jacobi-type matrix of order $\sigma$. In particular, we examine the case where $W_{\sigma}-W_{\sigma}^{0}$ is compact. Thus in what follows we assume that our sequences tend to the operator coefficients rapidly enough; that is, $a_{n}^{i} \rightarrow \omega_{i}, b_{n} \rightarrow 0$, as $n \rightarrow \pm \infty$. Then the essential spectrum $\varsigma_{\text {ess }}$ is given by $\varsigma_{\text {ess }}\left(W_{\sigma}\right)=\varsigma_{\text {ess }}\left(W_{\sigma}^{0}\right)=\left[-{ }^{2 \sigma} C_{\sigma}, 4^{\sigma}-{ }^{2 \sigma} C_{\sigma}\right]$ and $W_{\sigma}$ may have simple eigenvalues $\left\{E_{j}^{ \pm}\right\}_{j=1}^{N_{ \pm}}$where $N_{ \pm} \in \overline{\mathbb{N}}$, and

$$
E_{1}^{+}>E_{2}^{+}>\cdots>44^{\sigma}-{ }^{2 \sigma} C_{\sigma}>-{ }^{2 \sigma} C_{\sigma}>\cdots>E_{2}^{-}>E_{1}^{-} .
$$

Theorem 5. Let $\gamma \geq 1,\left\{b_{n}\right\}_{n \in \mathbb{Z}}$, and $\left\{a_{n}^{i}-\omega_{i}\right\}_{n \in \mathbb{Z}} \in \ell^{\gamma+1 / 2 \sigma}(\mathbb{Z})$ for all $i \in\{1, \ldots, \sigma\}$. Then for the eigenvalues $\left\{E_{j}^{ \pm}\right\}_{j=1}^{N_{ \pm}}$of the operator $W_{\sigma}$ we have

$$
\begin{aligned}
& \sum_{j=1}^{N_{-}}\left|E_{j}^{-}+{ }^{2 \sigma} C_{\sigma}\right|^{\gamma}+\sum_{j=1}^{N_{+}}\left|E_{j}^{+}-\left(4^{\sigma}-{ }^{2 \sigma} C_{\sigma}\right)\right|^{\gamma} \\
& \leq \nu_{\sigma}^{\gamma}\left(\sum_{n \in \mathbb{Z}}\left|b_{n}\right|^{\gamma+1 / 2 \sigma}+4 \sum_{n \in \mathbb{Z} k=1} \sum_{k=1}^{\sigma}\left|a_{n}^{k}-\omega_{k}\right|^{\gamma+1 / 2 \sigma}\right),
\end{aligned}
$$

where

$$
\nu_{\sigma}^{\gamma}=2 \sigma(2 \sigma+1)^{\gamma-2} \frac{\Gamma((4 \sigma+1) / 2 \sigma) \Gamma(\gamma+1)}{\Gamma(\gamma+(2 \sigma+1) / 2 \sigma)} .
$$

\section{Auxiliary Results}

We require the following discrete Kolmogorov-type inequality.

Lemma 6. For a sequence $\varphi \in \ell^{2}(\mathbb{Z})$, and for $n>k \geq 1$, we have the following inequality:

$$
\left\|D^{k} \varphi\right\|_{\ell^{2}(\mathbb{Z})} \leq\|\varphi\|_{\ell^{2}(\mathbb{Z})}^{1-k / n}\left\|D^{n} \varphi\right\|_{\ell^{2}(\mathbb{Z})}^{k / n} .
$$

Proof. We proceed by induction, where we note that the initial case, $k=1, n=2$, holds true as the inequality

$$
\|D \varphi\|_{\ell^{2}(\mathbb{Z})} \leq\|\varphi\|_{\ell^{2}(\mathbb{Z})}^{1 / 2}\left\|D^{2} \varphi\right\|_{\ell^{2}(\mathbb{Z})}^{1 / 2}
$$

is in fact the simple inequality found by Copson in [7]. This case in turn, if used repeatedly, shows that the inequality holds true for all $k$, if $n=k+1$. We then take the inductive step on the variable $n$. Hence we assume that we have the required inequality for $k<n \leq m$, given a fixed $k$, and proceed to prove the statement for $n=m+1$. Thus

$$
\begin{aligned}
\left\|D^{m} \varphi\right\|_{\ell^{2}(\mathbb{Z})}^{2} & =\left\langle D^{m} \varphi, D^{m} \varphi\right\rangle=\left\langle D^{*} D^{m} \varphi, D^{m-1} \varphi\right\rangle \\
& \leq\left\|D^{m+1} \varphi\right\|_{\ell^{2}(\mathbb{Z})}\left\|D^{m-1} \varphi\right\|_{\ell^{2}(\mathbb{Z})} .
\end{aligned}
$$

We thus apply our induction hypothesis and set $k=m-1$ and $n=m$ as follows:

$$
\begin{aligned}
\left\|D^{m} \varphi\right\|_{\ell^{2}(\mathbb{Z})}^{2} & \leq\left\|D^{m+1} \varphi\right\|_{\ell^{2}(\mathbb{Z})}\left\|D^{m} \varphi\right\|_{\ell^{2}(\mathbb{Z})}^{(m-1) / m}\|\varphi\|_{\ell^{2}(\mathbb{Z})}^{1 / m} \\
& \Longrightarrow\left\|D^{m} \varphi\right\|_{\ell^{2}(\mathbb{Z})} \leq\left\|D^{m+1} \varphi\right\|_{\ell^{2}(\mathbb{Z})}^{m /(m+1)}\|\varphi\|_{\ell^{2}(\mathbb{Z})}^{1 /(m+1)} .
\end{aligned}
$$

We now return to the induction hypothesis as follows:

$$
\begin{aligned}
\left\|D^{k} \varphi\right\|_{\ell^{2}(\mathbb{Z})} & \leq\left\|D^{m} \varphi\right\|_{\ell^{2}(\mathbb{Z})}^{k / m}\|\varphi\|_{\ell^{2}(\mathbb{Z})}^{(m-k) / m} \\
& \leq\left\|D^{m+1} \varphi\right\|_{\ell^{2}(\mathbb{Z})}^{k /(m+1)}\|\varphi\|_{\ell^{2}(\mathbb{Z})}^{k / m(m+1)}\|\varphi\|_{\ell^{2}(\mathbb{Z})}^{(m-k) / m} \\
& =\left\|D^{m+1} \varphi\right\|_{\ell^{2}(\mathbb{Z})}^{k /(m+1)}\|\varphi\|_{\ell^{2}(\mathbb{Z})}^{1-k /(m+1)} .
\end{aligned}
$$

We are now equipped to prove an Agmon-Kolmogorovtype inequality.

Proposition 7. For a sequence $\varphi \in \ell^{2}(\mathbb{Z})$, we have for any $\sigma \in \mathbb{N}$

$$
\|\varphi\|_{\ell^{\infty}(\mathbb{Z})} \leq\|\varphi\|_{\ell^{2}(\mathbb{Z})}^{1-1 / 2 \sigma}\left\|D^{\sigma} \varphi\right\|_{\ell^{2}(\mathbb{Z})}^{1 / 2 \sigma}
$$

Proof. First we use Lemma 6 with $k=1, n=\sigma$ as follows:

$$
\|D \varphi\|_{\ell^{2}(\mathbb{Z})} \leq\|\varphi\|_{\ell^{2}(\mathbb{Z})}^{1-1 / \sigma}\left\|D^{\sigma} \varphi\right\|_{\ell^{2}(\mathbb{Z})}^{1 / \sigma},
$$

and we apply this estimate to the well-known discrete Agmon inequality (see [4]):

$$
\begin{aligned}
|\varphi(n)|^{2} & \leq\|\varphi\|_{\ell^{2}(\mathbb{Z})}\|D \varphi\|_{\ell^{2}(\mathbb{Z})} \\
& \leq\|\varphi\|_{\ell^{2}(\mathbb{Z})}\|\varphi\|_{\ell^{2}(\mathbb{Z})}^{1-1 / \sigma}\left\|D^{\sigma} \varphi\right\|_{\ell^{2}(\mathbb{Z})}^{1 / \sigma} \\
& =\|\varphi\|_{\ell^{2}(\mathbb{Z})}^{2-1 / \sigma}\left\|D^{\sigma} \varphi\right\|_{\ell^{2}(\mathbb{Z})}^{1 / \sigma} .
\end{aligned}
$$

Proposition 8. Let $\left\{\psi_{j}\right\}_{j=1}^{N}$ be an orthonormal system of sequences in $\ell^{2}(\mathbb{Z}) ;$ that is, $\left\langle\psi_{j}, \psi_{k}\right\rangle=\delta_{j k}$, and let $\rho(n):=\sum_{j=1}^{N}$ $\left|\psi_{j}(n)\right|^{2}$. Then

$$
\sum_{n \in \mathbb{Z}} \rho^{2 \sigma+1}(n) \leq \sum_{j=1}^{N} \sum_{n \in \mathbb{Z}}\left|D^{\sigma} \psi_{j}(n)\right|^{2}
$$

Proof. Let $\xi=\left(\xi_{1}, \xi_{2}, \ldots, \xi_{N}\right) \in \mathbb{C}^{N}$. By Proposition 7 , we have

$$
\begin{aligned}
\left|\sum_{j=1}^{N} \xi_{j} \psi_{j}(n)\right|^{2} & \leq\left\|\sum_{j=1}^{N} \xi_{j} \psi_{j}\right\|_{\ell^{2}(\mathbb{Z})}^{(2 \sigma-1) / \sigma}\left\|D^{\sigma} \sum_{j=1}^{N} \xi_{j} \psi_{j}\right\|_{\ell^{2}(\mathbb{Z})}^{1 / \sigma} \\
& =\left(\sum_{j, k=1}^{N} \xi_{j} \bar{\xi}_{k}\left\langle\psi_{j}, \psi_{k}\right\rangle\right)^{(2 \sigma-1) / 2 \sigma}
\end{aligned}
$$




$$
\begin{aligned}
& \times\left(\sum_{j, k=1}^{N} \xi_{j} \bar{\xi}_{k}\left\langle D^{\sigma} \psi_{j}, D^{\sigma} \psi_{k}\right\rangle\right)^{1 / 2 \sigma} \\
\leq & \left(\sum_{j=1}^{N}\left|\xi_{j}\right|^{2}\right)^{(2 \sigma-1) / 2 \sigma} \\
& \times\left(\sum_{j, k=1}^{N} \xi_{j} \bar{\xi}_{k}\left\langle D^{\sigma} \psi_{j}, D^{\sigma} \psi_{k}\right\rangle\right)^{1 / 2 \sigma} .
\end{aligned}
$$

Let $\xi_{j}:=\overline{\psi_{j}(n)}$ and as $\rho(n)=\sum_{j=1}^{N}\left|\psi_{j}(n)\right|^{2}$,

$$
\begin{aligned}
\rho^{2}(n) \leq & \rho^{(2 \sigma-1) / 2 \sigma}(n) \\
& \times\left(\sum_{j, k=1}^{N} \psi_{j}(n) \overline{\psi_{k}(n)}\left\langle D^{\sigma} \psi_{j}, D^{\sigma} \psi_{k}\right\rangle\right)^{1 / 2 \sigma} \\
\Longrightarrow & \rho^{2 \sigma+1}(n) \leq \sum_{j, k=1}^{N} \psi_{j}(n) \overline{\psi_{k}(n)}\left\langle D^{\sigma} \psi_{j}, D^{\sigma} \psi_{k}\right\rangle \\
\Longrightarrow & \sum_{n \in \mathbb{Z}} \rho^{2 \sigma+1}(n) \leq \sum_{j=1}^{N}\left(\sum_{n \in \mathbb{Z}}\left|D^{\sigma} \psi_{j}(n)\right|^{2}\right) .
\end{aligned}
$$

\section{Proof of Theorem 2}

We take the inner product with $\psi_{j}(n)$ on (10) and sum both sides of the equation with respect to $j$. We obtain

$$
\sum_{j=1}^{N} e_{j}=\sum_{j=1}^{N}\left(\sum_{n \in \mathbb{Z}}\left|D^{\sigma} \psi_{j}(n)\right|^{2}\right)-\sum_{j=1}^{N}\left(\sum_{n \in \mathbb{Z}} b_{n}\left|\psi_{j}(n)\right|^{2}\right) .
$$

We now use Proposition 8 and apply the appropriate Hölder's inequality; that is,

$$
\begin{aligned}
\sum_{j=1}^{N} e_{j} \geq & \sum_{n \in \mathbb{Z}}\left(\sum_{j=1}^{N}\left|\psi_{j}(n)\right|^{2}\right)^{2 \sigma+1} \\
& -\left(\sum_{n \in \mathbb{Z}} b_{n}^{(2 \sigma+1) / 2 \sigma}\right)^{2 \sigma /(2 \sigma+1)} \\
& \times\left(\sum_{n \in \mathbb{Z}}\left(\sum_{j=1}^{N}\left|\psi_{j}(n)\right|^{2}\right)^{2 \sigma+1}\right)^{1 /(2 \sigma+1)} .
\end{aligned}
$$

We define

$$
\begin{gathered}
\chi:=\left(\sum_{n \in \mathbb{Z}}\left(\sum_{j=1}^{N}\left|\psi_{j}(n)\right|^{2}\right)^{2 \sigma+1}\right)^{1 /(2 \sigma+1)}, \\
\varsigma:=\left(\sum_{n \in \mathbb{Z}} b_{n}^{(2 \sigma+1) / 2 \sigma}\right)^{2 \sigma /(2 \sigma+1)} .
\end{gathered}
$$

The latter inequality can be written as

$$
\chi^{2 \sigma+1}-\varsigma \chi \leq \sum_{j=1}^{N} e_{j}
$$

The LHS is maximal when

$$
\chi=\frac{1}{(2 \sigma+1)^{1 / 2 \sigma}}\left(\sum_{n \in \mathbb{Z}} b_{n}^{(2 \sigma+1) / 2 \sigma}\right)^{1 /(2 \sigma+1)} .
$$

Substituting this into (33), we obtain

$$
\begin{aligned}
\sum_{j=1}^{N} e_{j} \geq & \left(\frac{1}{(2 \sigma+1)^{(2 \sigma+1) / 2 \sigma}} \sum_{n \in \mathbb{Z}} b_{n}^{(2 \sigma+1) / 2 \sigma}\right) \\
& -\frac{1}{(2 \sigma+1)^{1 / 2 \sigma}} \sum_{n \in \mathbb{Z}} b_{n}^{(2 \sigma+1) / 2 \sigma} . \\
= & \frac{-2 \sigma}{(2 \sigma+1)^{(2 \sigma+1) / 2 \sigma}} \sum_{n \in \mathbb{Z}} b_{n}^{(2 \sigma+1) / 2 \sigma} .
\end{aligned}
$$

Therefore,

$$
\sum_{j=1}^{N}\left|e_{j}\right| \leq \frac{2 \sigma}{(2 \sigma+1)^{(2 \sigma+1) / 2 \sigma}} \sum_{n \in \mathbb{Z}} b_{n}^{(2 \sigma+1) / 2 \sigma} .
$$

We lift this bound now with regard to moments by using the standard Aizenman-Lieb procedure (see [8]). We let $\left\{e_{j}(\tau)\right\}_{j=1}^{N}$ be the negative eigenvalues of the operator $\Delta_{D}^{\sigma}-$ $\left(b_{n}-\tau\right)_{+}$. By the variational principle for the negative eigenvalues $\left\{-\left(\left|e_{j}\right|-\tau\right)_{+}\right\}_{j=1}^{N}$ of the operator $\Delta_{D}^{\sigma}-\left(b_{n}-\tau\right)$ we have

$$
\left(\left|e_{j}\right|-\tau\right)_{+} \leq\left|e_{j}(\tau)\right| .
$$

By this estimate, we find that

$$
\begin{aligned}
\sum_{j=1}^{N}\left|e_{j}\right|^{\gamma}= & \frac{1}{\mathscr{B}(\gamma-1,2)} \int_{0}^{\infty} \tau^{\gamma-2} \sum_{j=1}^{N}\left(\left|e_{j}\right|-\tau\right)_{+} d \tau \\
\leq & \frac{1}{\mathscr{B}(\gamma-1,2)} \int_{0}^{\infty} \tau^{\gamma-2} \sum_{j=1}^{N}\left|e_{j}(\tau)\right| d \tau \\
\leq & \frac{2 \sigma}{(2 \sigma+1)^{(2 \sigma+1) / 2 \sigma}} \frac{1}{\mathscr{B}(\gamma-1,2)} \\
& \times \int_{0}^{\infty} \tau^{\gamma-2} \sum_{n \in \mathbb{Z}}\left(b_{n}-\tau\right)_{+}^{(2 \sigma+1) / 2 \sigma} d \tau,
\end{aligned}
$$

by (38) above, where $B(x, y)=\Gamma(x) \Gamma(y) / \Gamma(x+y)$ is the wellknown Beta function. Thus, after a change of variable,

$$
\begin{aligned}
\sum_{j=1}^{N}\left|e_{j}\right|^{\gamma} \leq & \frac{2 \sigma}{(2 \sigma+1)^{(2 \sigma+1) / 2 \sigma}} \\
& \times \frac{\Gamma((4 \sigma+1) / 2 \sigma) \Gamma(\gamma+1)}{\Gamma(\gamma+(2 \sigma+1) / 2 \sigma)} \sum_{n \in \mathbb{Z}} b_{n}^{\gamma+1 / 2 \sigma},
\end{aligned}
$$

completing our proof. 


\section{Proof of Theorem 5}

We have the following matrix bounds for square, $m \times m$ matrices, as given in [2]. For $a_{n}^{m}, \omega_{m} \in \mathbb{R}$, we have

$$
\begin{aligned}
& \left(\begin{array}{ccccc}
-\left|a_{n}^{m}-\omega_{m}\right| & 0 & \ldots & 0 & \omega_{m} \\
0 & 0 & \ldots & 0 & 0 \\
\vdots & \vdots & \ddots & \vdots & \vdots \\
0 & 0 & \ldots & 0 & 0 \\
\omega_{m} & 0 & \ldots & 0 & -\left|a_{n}^{m}-\omega_{m}\right|
\end{array}\right) \\
& \leq\left(\begin{array}{ccccc}
0 & 0 & \ldots & 0 & a_{n}^{m} \\
0 & 0 & \ldots & 0 & 0 \\
\vdots & \vdots & \ddots & \vdots & \vdots \\
0 & 0 & \ldots & 0 & 0 \\
a_{n}^{m} & 0 & \ldots & 0 & 0
\end{array}\right) \\
& \leq\left(\begin{array}{ccccc}
\left|a_{n}^{m}-\omega_{m}\right| & 0 & \ldots & 0 & \omega_{m} \\
0 & 0 & \ldots & 0 & 0 \\
\vdots & \vdots & \ddots & \vdots & \vdots \\
0 & 0 & \ldots & 0 & 0 \\
\omega_{m} & 0 & \ldots & 0 & \left|a_{n}^{m}-\omega_{m}\right|
\end{array}\right) \text {. }
\end{aligned}
$$

We thus use this on each block of indices of $W_{\sigma}$ as follows:

$$
\begin{aligned}
& W_{\sigma}\left(\left\{a_{n}^{1} \equiv \omega_{1}\right\}, \ldots,\left\{a_{n}^{\sigma} \equiv \omega_{\sigma}\right\},\left\{b_{n}^{(-)}\right\}\right) \\
& \quad \leq W_{\sigma}\left(\left\{a_{n}^{1}\right\}, \ldots,\left\{a_{n}^{\sigma}\right\},\left\{b_{n}\right\}\right) \\
& \quad \leq W_{\sigma}\left(\left\{a_{n}^{1} \equiv \omega_{1}\right\}, \ldots,\left\{a_{n}^{\sigma} \equiv \omega_{\sigma}\right\},\left\{b_{n}^{(+)}\right\}\right),
\end{aligned}
$$

where $b_{n}^{( \pm)}$is given by

$$
\begin{aligned}
b_{n}^{( \pm)}=b_{n} \pm & \left(\left(\left|a_{n-1}^{1}-\omega_{1}\right|+\left|a_{n}^{1}-\omega_{1}\right|\right)\right. \\
& \left.+\cdots+\left(\left|a_{n-\sigma}^{\sigma}-\omega_{\sigma}\right|+\left|a_{n}^{\sigma}-\omega_{\sigma}\right|\right)\right)
\end{aligned}
$$

that is,

$$
b_{n}^{( \pm)}=b_{n} \pm\left(\sum_{k=1}^{\sigma}\left|a_{n-k}^{k}-\omega_{k}\right|+\left|a_{n}^{k}-\omega_{k}\right|\right) \text {. }
$$

Now we relate these to our Schrödinger-type operators:

$$
\begin{gathered}
\Delta_{D}^{\sigma}-4^{\sigma}+b_{n}=W_{\sigma}^{0}-\left(4^{\sigma}-{ }^{2 \sigma} C_{\sigma}\right)+b_{n} \\
=W_{\sigma}\left(\left\{a_{n}^{1} \equiv \omega_{1}\right\}, \ldots,\left\{a_{n}^{\sigma} \equiv \omega_{\sigma}\right\},\right. \\
\left.\quad\left\{b_{n}-\left(4^{\sigma}-{ }^{2 \sigma} C_{\sigma}\right)\right\}\right), \\
\Delta_{D}^{\sigma}+b_{n}=W_{\sigma}^{0}+{ }^{2 \sigma} C_{\sigma}+b_{n} \\
=W_{\sigma}\left(\left\{a_{n}^{1} \equiv \omega_{1}\right\}, \ldots,\left\{a_{n}^{\sigma} \equiv \omega_{\sigma}\right\},\right. \\
\left.\quad\left\{b_{n}+{ }^{2 \sigma} C_{\sigma}\right\}\right) .
\end{gathered}
$$

Now $\left(E_{j}^{+}-\left(4^{\sigma}-{ }^{2 \sigma} C_{\sigma}\right)\right)$ are positive eigenvalues of $W_{\sigma}\left(\left\{a_{n}^{1}\right\}, \ldots\right.$, $\left.\left\{a_{n}^{\sigma}\right\},\left\{b_{n}-\left(4^{\sigma}-{ }^{2 \sigma} C_{\sigma}\right)\right\}\right)$. Thus by using (43) and the variational principle, we have

$$
\begin{aligned}
& W_{\sigma}\left(\left\{a_{n}^{1}\right\}, \ldots,\left\{a_{n}^{\sigma}\right\},\left\{b_{n}-\left(4^{\sigma}-{ }^{2 \sigma} C_{\sigma}\right)\right\}\right) \\
& \quad \leq W_{\sigma}\left(\left\{a_{n}^{1} \equiv \omega_{1}\right\}, \ldots,\left\{a_{n}^{\sigma} \equiv \omega_{\sigma}\right\},\left\{b_{n}^{(+)}-\left(4^{\sigma}-{ }^{2 \sigma} C_{\sigma}\right)\right\}\right), \\
& \Longrightarrow\left|E_{j}^{+}-\left(4^{\sigma}-{ }^{2 \sigma} C_{\sigma}\right)\right| \leq e_{j}^{+},
\end{aligned}
$$

where $e_{j}^{+}$are the positive eigenvalues of

$$
\begin{aligned}
& W_{\sigma}\left(\left\{a_{n}^{1} \equiv \omega_{1}\right\}, \ldots,\left\{a_{n}^{\sigma} \equiv \omega_{\sigma}\right\},\left\{b_{n}^{(+)}-\left(4^{\sigma}-{ }^{2 \sigma} C_{\sigma}\right)\right\}\right) \\
& \quad=\Delta_{D}^{\sigma}-4^{\sigma}+b_{n}^{(+)} .
\end{aligned}
$$

Let us now define $\left(b_{n}\right)_{+}:=\max \left(b_{n}, 0\right),\left(b_{n}\right)_{-}:=-\min \left(b_{n}, 0\right)$. Then, by Corollary 4 for the positive eigenvalues of our operator, we have

$$
\sum_{j=1}^{N_{+}}\left(e_{j}^{+}\right)^{\gamma} \leq \eta_{\sigma}^{\gamma} \sum_{n \in \mathbb{Z}}\left(b_{n}^{(+)}\right)_{+}^{\gamma+1 / 2 \sigma}
$$

Thus, applying (48),

$$
\begin{aligned}
& \sum_{j=1}^{N_{+}}\left|E_{j}^{+}-\left(4^{\sigma}-{ }^{2 \sigma} C_{\sigma}\right)\right|^{\gamma} \\
& \quad \leq \eta_{\sigma}^{\gamma} \sum_{n \in \mathbb{Z}}\left(\left(b_{n}\right)_{+}+\sum_{k=1}^{\sigma}\left(\left|a_{n-k}^{k}-\omega_{k}\right|+\left|a_{n}^{k}-\omega_{k}\right|\right)\right)^{\gamma+1 / 2 \sigma},
\end{aligned}
$$

where

$$
\eta_{\sigma}^{\gamma}:=\frac{2 \sigma}{(2 \sigma+1)^{(2 \sigma+1) / 2 \sigma}} \frac{\Gamma((4 \sigma+1) / 2 \sigma) \Gamma(\gamma+1)}{\Gamma(\gamma+(2 \sigma+1) / 2 \sigma)} .
$$

Similarly, using Theorem 2 on (47),

$$
\begin{aligned}
& \sum_{j=1}^{N_{-}}\left|E_{j}^{-}+{ }^{2 \sigma} C_{\sigma}\right|^{\gamma} \\
& \quad \leq \eta_{\sigma}^{\gamma} \sum_{n \in \mathbb{Z}}\left(\left(b_{n}\right)_{-}+\sum_{k=1}^{\sigma}\left(\left|a_{n-k}^{k}-\omega_{k}\right|+\left|a_{n}^{k}-\omega_{k}\right|\right)\right)^{\gamma+1 / 2 \sigma}
\end{aligned}
$$

Using the following application of Jensen's inequality, that is, for $i \in\{1, \ldots, 2 \sigma+1\}$, let $\alpha_{i}, q \in \mathbb{R}$, with $q \geq 1$,

$$
\left(\sum_{i=1}^{2 \sigma+1} \alpha_{i}\right)^{q} \leq(2 \sigma+1)^{q-1}\left(\sum_{i=1}^{2 \sigma+1} \alpha_{i}^{q}\right)
$$


to each of (51) and (53), we have

$$
\begin{gathered}
\left(\left(b_{n}\right)_{ \pm}+\sum_{k=1}^{\sigma}\left(\left|a_{n-k}^{k}-\omega_{k}\right|+\left|a_{n}^{k}-\omega_{k}\right|\right)\right)^{\gamma+1 / 2 \sigma} \\
\leq(2 \sigma+1)^{\gamma-(2 \sigma-1) / 2 \sigma} \\
\times\left(\left(b_{n}\right)_{ \pm}^{\gamma+1 / 2 \sigma}+\sum_{k=1}^{\sigma}\left(\left|a_{n-k}^{k}-\omega_{k}\right|^{\gamma+1 / 2 \sigma}\right.\right. \\
\left.\left.+\left|a_{n}^{k}-\omega_{k}\right|^{\gamma+1 / 2 \sigma}\right)\right) .
\end{gathered}
$$

Summing these two inequalities, we arrive at

$$
\begin{aligned}
& \sum_{j=1}^{N_{-}}\left|E_{j}^{-}+{ }^{2 \sigma} C_{\sigma}\right|^{\gamma}+\sum_{j=1}^{N_{+}}\left|E_{j}^{+}-\left(4^{\sigma}-{ }^{2 \sigma} C_{\sigma}\right)\right|^{\gamma} \\
& \leq \nu_{\sigma}^{\gamma}\left(\sum_{n \in \mathbb{Z}}\left|b_{n}\right|^{\gamma+1 / 2 \sigma}+4 \sum_{n \in \mathbb{Z}} \sum_{k=1}^{\sigma}\left|a_{n}^{k}-\omega_{k}\right|^{\gamma+1 / 2 \sigma}\right),
\end{aligned}
$$

where

$$
\nu_{\sigma}^{\gamma}=2 \sigma(2 \sigma+1)^{\gamma-2} \frac{\Gamma((4 \sigma+1) / 2 \sigma) \Gamma(\gamma+1)}{\Gamma(\gamma+(2 \sigma+1) / 2 \sigma)},
$$

and the proof of Theorem 5 is complete.

\section{Conflict of Interests}

The author declares that there is no conflict of interests regarding the publication of this paper.

\section{References}

[1] R. Killip and B. Simon, "Sum rules for Jacobi matrices and their applications to spectral theory," Annals of Mathematics, vol. 158, no. 1, pp. 253-321, 2003.

[2] D. Hundertmark and B. Simon, "Lieb-thirring inequalities for Jacobi matrices," Journal of Approximation Theory, vol. 118, no. 1, pp. 106-130, 2002.

[3] D. Bakić and B. Guljaš, "A note on compact operators and operator matrices," Mathematical Communications, vol. 4, no. 2, pp. 159-165, 1999.

[4] A. Sahovic, "New constants in discrete Lieb-Thirring inequalities for Jacobi matrices," Journal of Mathematical Sciences, vol. 166, no. 3, pp. 319-327, 2010.

[5] J. Dolbeault, A. Laptev, and M. Loss, "Lieb-Thirring inequalities with improved constants," Journal of the European Mathematical Society, vol. 10, no. 4, pp. 1121-1126, 2008.

[6] A. Eden and C. Foias, "A simple proof of the generalized Lieb-Thirring inequalities in one-space dimension," Journal of Mathematical Analysis and Applications, vol. 162, no. 1, pp. 250254, 1991.

[7] E. T. Copson, "Two series inequalities," Proceedings of the Royal Society of Edinburgh, Section A, vol. 83, no. 1-2, pp. 109-114, 1979.

[8] M. Aizenmann and E. H. Lieb, "On semi-classical bound for eigenvalues of Schrödinger operators," Physics Letters A, vol. 66, no. 6, pp. 427-429, 1978. 


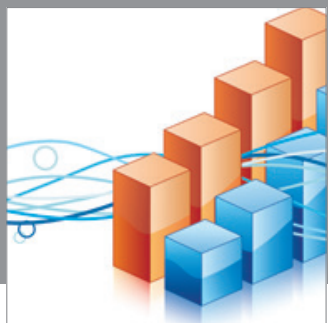

Advances in

Operations Research

mansans

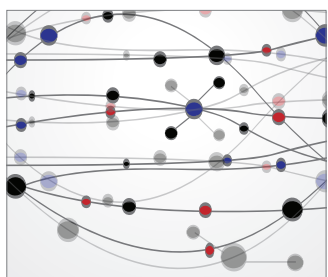

The Scientific World Journal
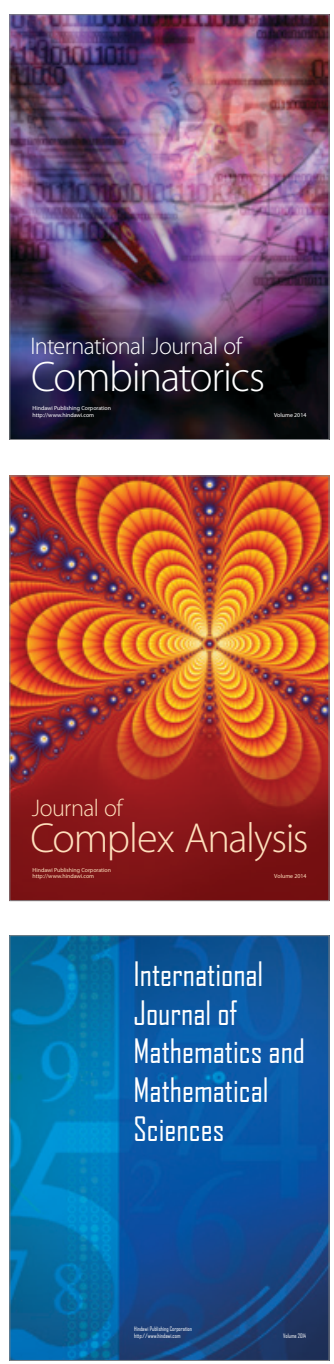
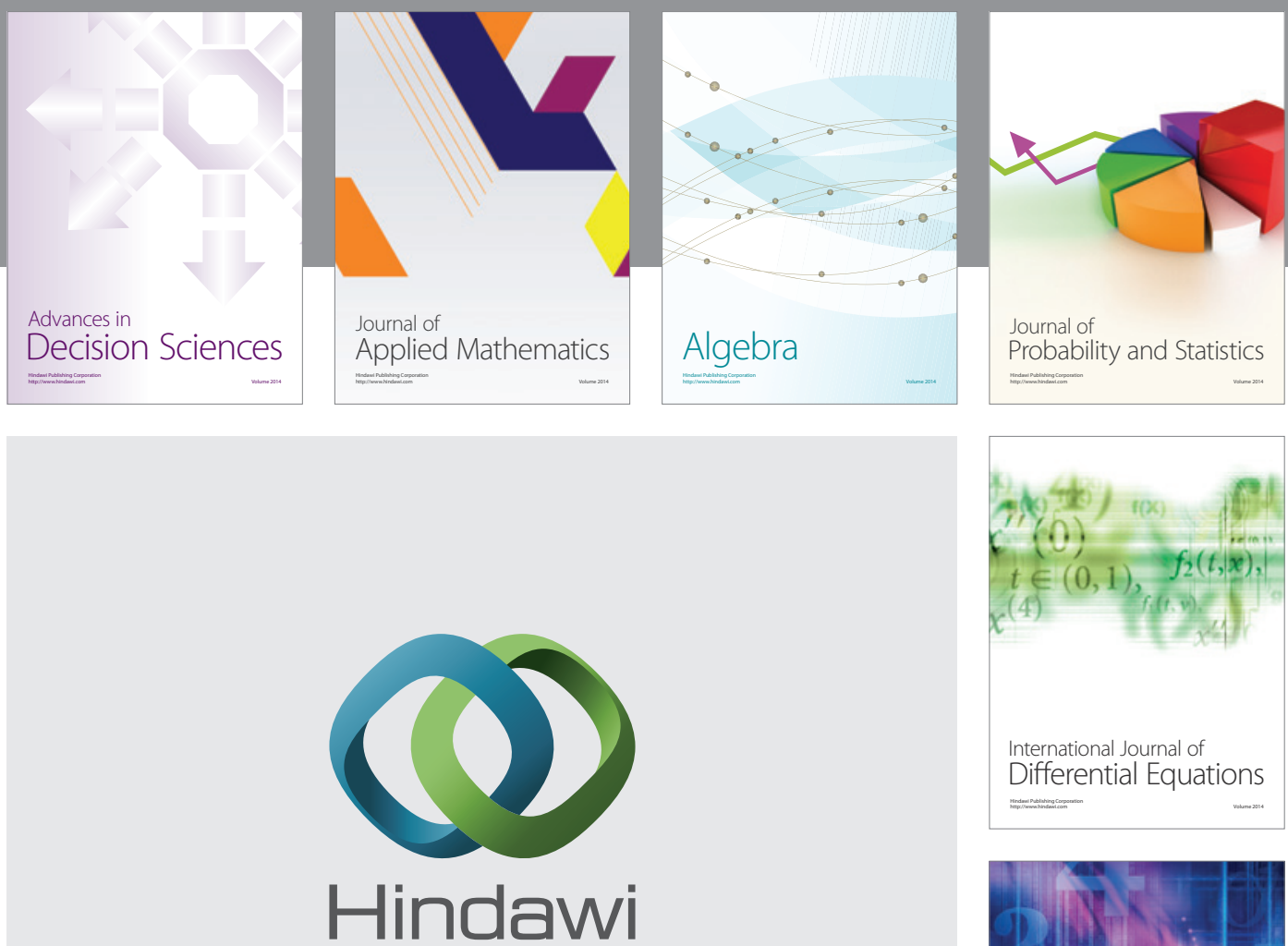

Submit your manuscripts at http://www.hindawi.com
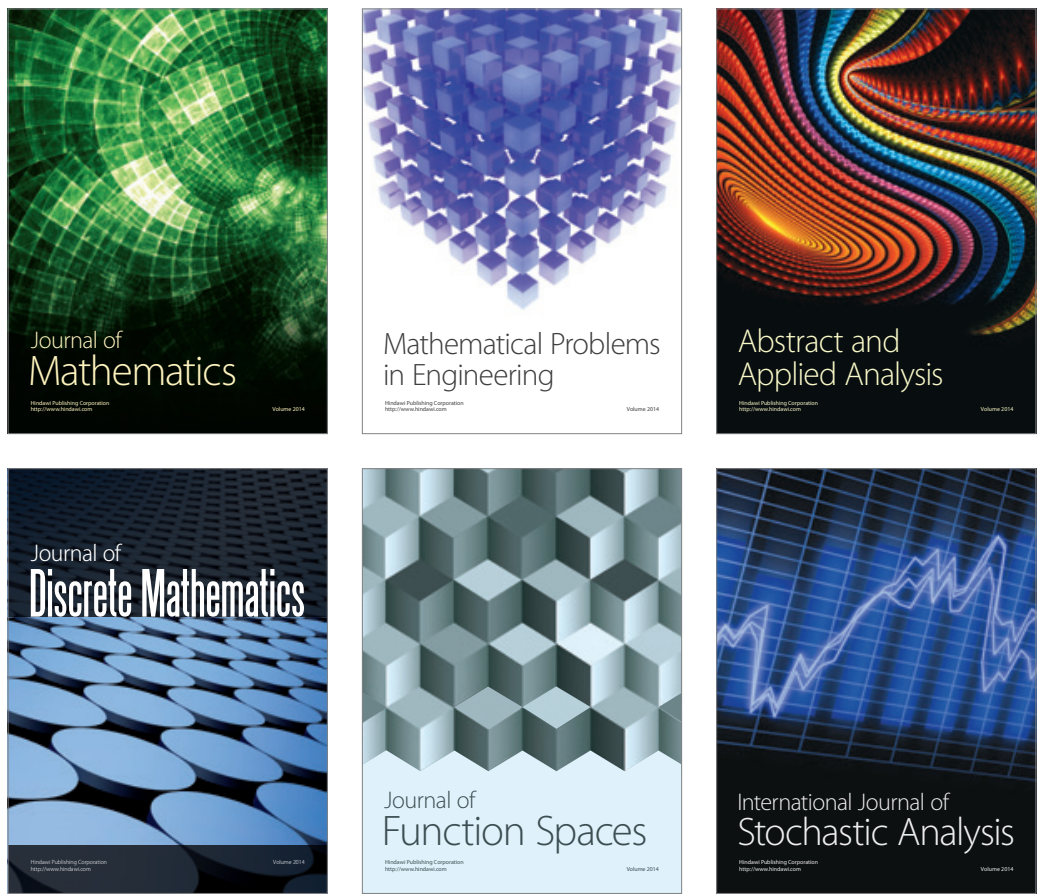

Journal of

Function Spaces

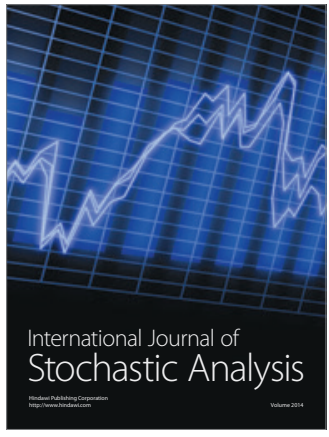

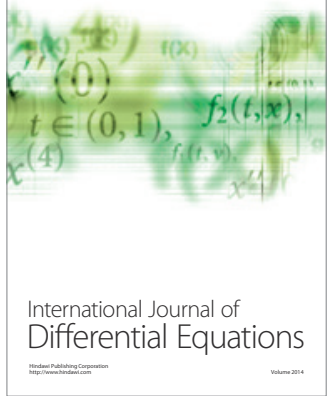
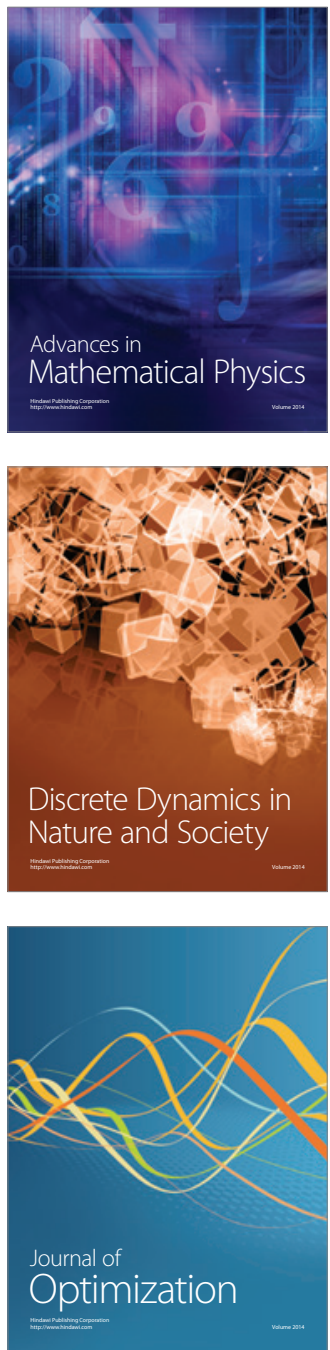